\title{
Histological Changes in Renal, Hepatic and Cardiac Tissues of Wistar Rats after 6 Weeks Treatment with Bipyridine Gold (III) Complex with Dithiocarbamate Ligands
}

\author{
Ahmed Badar 1,*(D), Ayesha Ahmed ${ }^{2} \mathbb{D}$, Dalal M. Al-Tamimi ${ }^{2}$, Anvarhusein A. Isab ${ }^{3}$, Muhammad Altaf 4 (D) \\ and Sania Ahmed ${ }^{5}$ \\ 1 Department of Physiology, College of Medicine, Imam Abdulrahman Bin Faisal \\ University, Dammam 31441, Saudi Arabia \\ 2 Department of Pathology, College of Medicine, Imam Abdulrahman Bin Faisal University, \\ Dammam 31441, Saudi Arabia; aahmed@iau.edu.sa (A.A.); dtamimi@iau.edu.sa (D.M.A.-T.) \\ 3 Department of Chemistry, King Fahd University of Petroleum and Minerals, Dhahran 31261, Saudi Arabia; \\ aisab@kfupm.edu.sa \\ 4 Department of Chemistry, Government College University, Lahore 54000, Pakistan; \\ Muhammad.altaf@gcu.edu.pk \\ 5 Army Medical College, Abid Majeed Road, Rawalpindi 46000, Pakistan; saniaahmed95@hotmail.com \\ * Correspondence: absheikh@iau.edu.sa
}

Citation: Badar, A.; Ahmed, A.; Al-Tamimi, D.M.; Isab, A.A.; Altaf, M.; Ahmed, S. Histological Changes in Renal, Hepatic and Cardiac Tissues of Wistar Rats after 6 Weeks Treatment with Bipyridine Gold (III) Complex with Dithiocarbamate Ligands. Pharmaceutics 2021, 13, 1530. https:// doi.org/10.3390/pharmaceutics13101530

Academic Editors: Vanesa

Fernández-Moreira and

Elena Cerrada

Received: 31 July 2021

Accepted: 20 September 2021

Published: 23 September 2021

Publisher's Note: MDPI stays neutral with regard to jurisdictional claims in published maps and institutional affiliations.

Copyright: (c) 2021 by the authors Licensee MDPI, Basel, Switzerland. This article is an open access article distributed under the terms and conditions of the Creative Commons Attribution (CC BY) license (https:// creativecommons.org/licenses/by/ $4.0 /)$

\begin{abstract}
Bipyridine gold (III) dithiocarbamate compounds are Gold-III complexes with promising cytotoxic properties. In this study, the subacute toxicity of a Gold (III) complex with dithiocarbamate ligand was evaluated. In the acute toxicity component, an initial $\mathrm{LD}_{50}(38.46 \mathrm{mg} / \mathrm{kg})$ was calculated by the administration of 50,100, 200, 400, and $800 \mathrm{mg} / \mathrm{kg}$ of the compound to five groups of rats, respectively ( $n=4$ each). The sixth group was the control. The sub-acute toxicity component comprised the control group $\mathrm{A}(n=6)$ and the study groups $\mathrm{B}(n=10)$ and $\mathrm{C}(n=4)$, which were administered $1 \mathrm{~mL}$ distilled water, $1 / 10 \mathrm{LD}_{50}(3.8 \mathrm{mg} / \mathrm{kg})$, and $1 / 5 \mathrm{LD}_{50}(7.6 \mathrm{mg} / \mathrm{kg})$, respectively, daily for 6 weeks. The alive animals were then sacrificed. Autopsy; preservation of renal, hepatic and cardiac tissue in buffered formalin; histopathological processing; microscopic evaluation; and comparison with the controls were sequentially conducted. In the subacute toxicity study at dosages of $3.8 \mathrm{mg} / \mathrm{kg}$ and $7.6 \mathrm{mg} / \mathrm{kg}$, the renal tubules remained unaffected with no necrosis or vacuolization. Mild to moderate renal interstitial, hepatic capsular, lobular and portal inflammation along with mild focal hepatic vacuolization were present. At $3.8 \mathrm{mg} / \mathrm{kg}$, the cardiac muscle fibers were unremarkable in $80 \%(n=8)$ of the specimens, with mild focal hyalinization in $20 \%(n=2)$ of the specimens. The same was observed in $50 \%(n=2)$ of the specimens at $7.6 \mathrm{mg} / \mathrm{kg}$. Variable congestion was evident in all of the groups. In the subacute toxicity study, the absence of renal tubular necrosis or vacuolization, the presence of mild inflammatory hepatic and renal alterations, and predominantly unremarkable cardiac muscle fibers suggest that Bipyridine gold (III)-dithiocarbamate is safe in animal studies and is a potential candidate for clinical trials.
\end{abstract}

Keywords: gold-III compound; bipyridine gold (III)-dithiocarbamate; subacute toxicity; cardiotoxicity; nephrotoxicity; hepatotoxicity

\section{Introduction}

The utilization of metal-based compounds for treating cancer started when cisplatin (cis-dichlorodiammine platinum-II) was accidently discovered in the mid-1960s. A long era of development and the utilization of platinum-based drugs started with this discovery. These drugs had very strong anticancerous potential and proved to have a high merit as effective treatment modalities. Their use, however, was hampered by associated tumor resistance and side effects such as nephrotoxicity, hepatotoxicity, neurotoxicity, ototoxicity, myelosuppression, nausea, and dermatological complications [1,2]. This led to research 
that emphasized formulating and testing alternative metal-based drugs. The present anticancerous medicinal chemistry and drug design aims towards the formulation of novel metal-based organic complexes and compounds with a different pharmacological profile than traditional platinum-based drugs. Due to the effectiveness of cisplatin, the basic concept of developing drugs that are structurally analogous to cisplatin but with different metals was conceived. Structural analogy could preserve anticancer potential in the same way as a platinum compound while the variation in metallic nidus could diminish the platinum-associated adverse side effects.

The medical community has been familiar with the use of gold for a long time. This makes gold complexes readily acceptable. There is evidence that some gold complexes might be useful in cisplatin-resistant tumor cells. Mechanistic studies have reflected that the gold complexes do not interact with DNA as strongly as the platinum complexes do. This points to a different mechanism for cytotoxicity, such as thioredoxin reductase (TrxR) inhibition, COX-1/2 inhibition, DNA damage, cell cycle arrest, ROS accumulation, apoptosis induction, mitochondrial membrane potential changes, angiogenesis inhibition, cellular respiratory inhibition, etc. The proteomic results suggest that gold complexes could affect protein synthesis, energy metabolism, oxidative stress response, and the cytoskeleton. In addition, they may help in the splicing, trafficking, and stability of mRNA [3].

In addition to the antiproliferative properties, the main hinderances to drug development related to gold complexes are stability in the biological media, drug distribution, and target selectivity. This makes complexes with the most suitable ancillary ligands frontrunners. Ligands such as aminophosphines, phosphines, N-heterocyclic carbene, among many others, help transport of the complex in the blood and move across cell membranes to reach the target cells. Some gold complexes such as auranofin are known to have significant antitumor activity and low toxicity. However, some of them produce bioactive metabolites. In fact, this stopped the further development of many gold complexes after initial success [4].

Over the last few years, there have been many studies on the anticancer potential of a number of gold (I) and gold (III) complexes, in particular alkynyl gold derivatives. The water-solubility of phosphine ligands and the good aqueous solution stability of a few heterobimetallic alkynyl gold (I) complexes and trivalent gold (III) alkynyl complexes have made them easy chemicals to work with. Almost all of these studies are in vitro, and despite evidence of encouraging antiproliferative activity and acceptable toxicity profiles, no related complexes have entered clinical trials [5].

With their intrinsic, highly potent tumor anti proliferative potential, gold (III) complexes have recently attained an important role. Their mechanism of action is different from cisplatin [6], but they are electronically and structurally homogenous to platinum (II) compounds [7]. The actual breakthrough for the use of gold compounds will come when these compounds are designed to be more clinically effective and potent, with enhanced cancer cell specificity and selectivity along with diminished organ toxicity [1].

There are limited reports available in the literature that highlight the "in vivo" anticancer potential and cytotoxic characteristics of gold (III) compounds [8-10]. Our study group has previously designed and reported on the gold (III) compound [ $\mathrm{Au}\left(\right.$ (ethylenediamine) $\left.\mathrm{Cl}_{2}\right] \mathrm{Cl}$ and [trans- $\left.( \pm)-1,2-(\mathrm{DACH})_{2} \mathrm{Au}\right] \mathrm{Cl}_{3}$, where trans- $( \pm)-1,2-(\mathrm{DACH})$ is Bis(trans-1,2-Diaminocyclohexane), which has negligible renal and hepatic organ toxicity compared to other clinically prevalent anticancerous drugs [11].

Gold (III)-dithiocarbamate compounds are a new addition to this evolving series of gold III complexes with new additives. These also have strong anticancer potential, which is inherent to gold III metal containing compounds. These have shown promising anticancer properties involving a different mechanism of action than cisplatin both "in vivo" and "in vitro" [12]. However, studies regarding its organ toxicity are very limited.

In the "in vitro" component of this study, eight new bipyridine gold (III) dithiocarbamatecontaining complexes (Figure 1) were synthesized and characterized [13]. These were analyzed and tested in a set of cancer stem cell lines in "in vitro" studies. Compounds 1, 2, 4, 
5, 7, and 8 (Figure 1) were seen to have maximum cytotoxicity in Hodgkin lymphoma cells and in ovary, breast, and prostate cancer cell lines. The minimum inhibitory concentration $\left(\mathrm{IC}_{50}\right)$ was seen to be lower than the reference drug cisplatin. Compound 1 (Figures 1 and 2) was observed to be the most active compound in the cisplatin resistant cancer stem lines of ovary and breast cancer, designated as A2780cis and 2780CP-16, respectively [13]. In the current study we evaluated the renal, hepatic, and cardiotoxicity of Compound 1 (bipyridine gold (III) dithiocarbamate-containing complex) in rats who had been administered this compound, with the aim of assessing its vital organ toxicity profile in the context of a new preclinical study for drug development.<smiles></smiles><smiles></smiles>

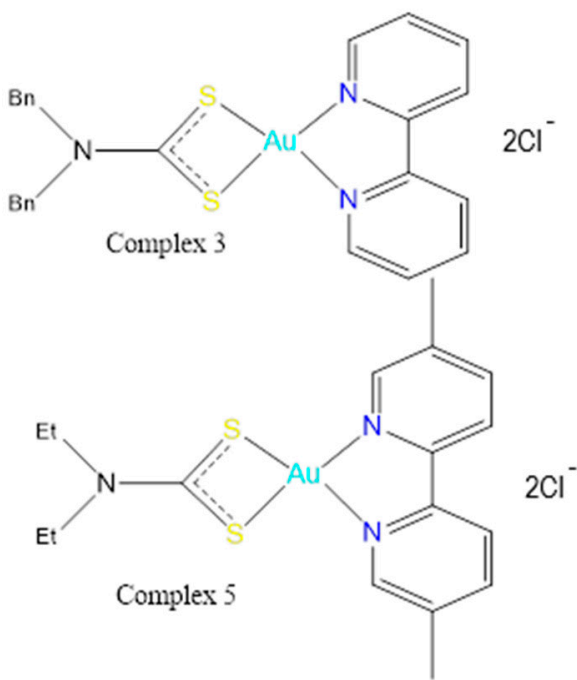

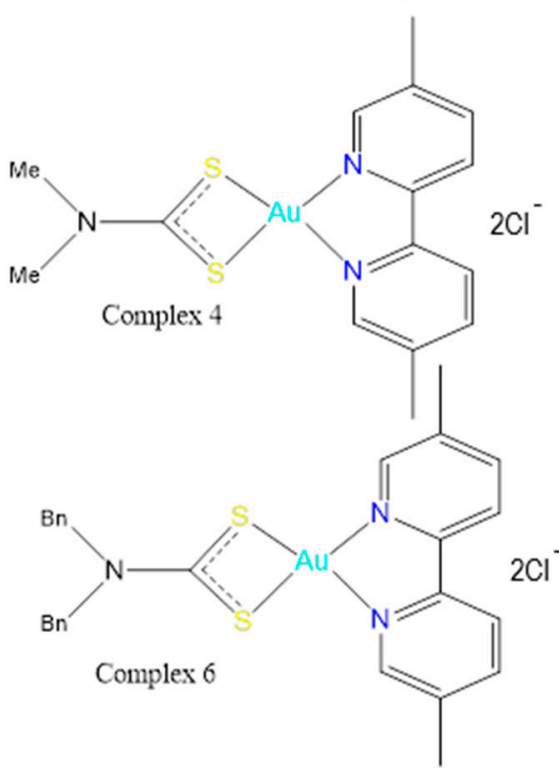<smiles></smiles><smiles></smiles>

Me: Methyl, Et: Ethyl, and Bn: Benzyl

Figure 1. The bipyridine gold (III) dithiocarbamate-containing complexes (1-8). 


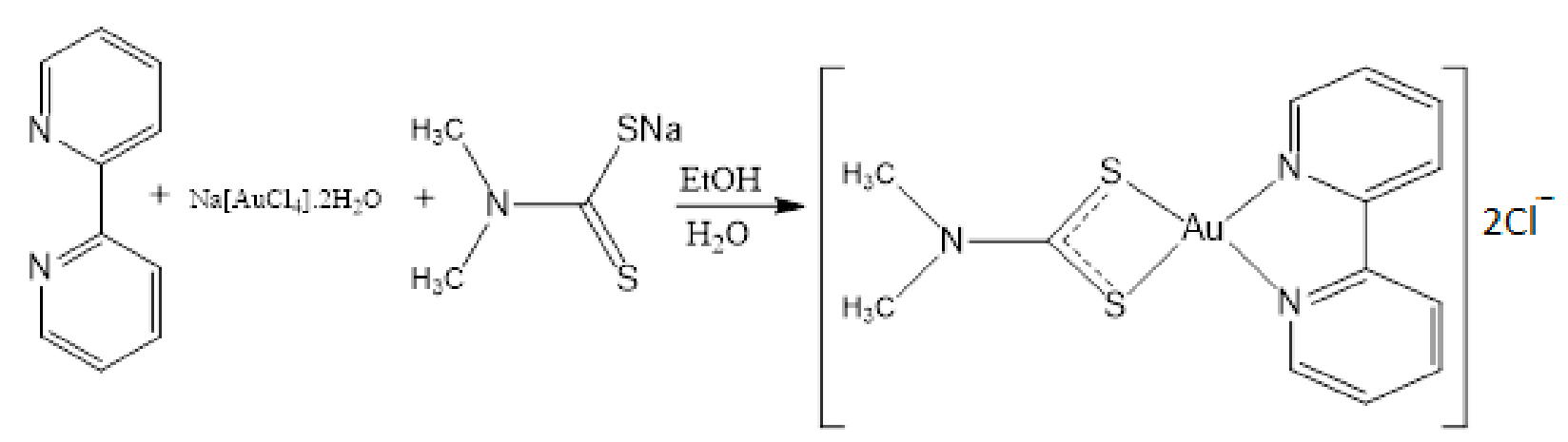

Figure 2. The synthesis of Complex (1).

\section{Materials and Methods}

This study was conducted at the College of Medicine, Imam Abdulrahman bin Faisal University (IAU), Dammam, Saudi Arabia, in collaboration with the King Fahd University of Petroleum and Minerals (KFUPM), Dhahran, Saudi Arabia (No: IN171005, dated 15 April 2018). The study was conducted following the OECD (Organization for Economic Co-operation and Development) principles of good laboratory practice [14] and was approved by the Institutional Review Board of Imam Abdulrahman Bin Faisal University (formerly the University of Dammam), Saudi Arabia. The acute as well as subacute toxicity study was conducted on Albino Wistar rats obtained from the animal house of IAU. We ensured the use of the minimal number of animals and euthanasia at predetermined end points to minimize the suffering of the animals.

An equal number of male and female rats $(n=50)$, weighing between 200-300 g, were obtained from animal house of Prince Muhammad Research center at Imam Abdulrahman University. The animals were kept in individual cages with standardized room temperature and humidity with free access to food and water for 7 days before the experiment.

\subsection{Syntheis of Complex (1)}

The complex (1) $[\mathrm{Au}(\mathrm{BPY})(\mathrm{DMTC})] \mathrm{Cl}_{2}(1)$ was synthesized by stepwise synthesis (Figure 2). Na[AuCl 4$] \cdot 2 \mathrm{H}_{2} \mathrm{O}, 0.5 \mathrm{mM}(200 \mathrm{mg}$ ) and 2,2'-Bipyridine $0.5 \mathrm{mM}$ (78 mg) were added simultaneously in $20 \mathrm{~mL}$ of ethanol, and the mixture was stirred for $3 \mathrm{~h}$ at room temperature. The sodium dimethyldithiocarbamate dihydrate $0.5 \mathrm{mM}(72 \mathrm{mg})$ in $10 \mathrm{~mL}$ distilled water was added slowly in the pale-yellow turbid solution obtained from the first step. The reaction mixture was stirred for an additional $1 \mathrm{~h}$ at room temperature. The final product appeared as a light-yellow precipitate in the solution. The precipitate was collected by filtration, washed with fresh distilled water $(3 \times 10 \mathrm{~mL})$, and dried at room temperature under vacuum. Yield: $83.09 \%(219.98 \mathrm{mg})$. FT-IR $\left(\mathrm{KBr}, v_{\max }, \mathrm{cm}^{-1}\right): 3570(\mathrm{~m})$, $3045(\mathrm{w}), 2926(\mathrm{w}) ; 1586(\mathrm{~m}), 1482(\mathrm{~s}), 1242(\mathrm{~m}), 1159(\mathrm{w}), 1039(\mathrm{~m}), 993(\mathrm{~m}), 967(\mathrm{w}), 761(\mathrm{~s})$, $565(\mathrm{~m}), 439(\mathrm{~m}) .{ }^{1} \mathrm{H}$ NMR $\left(500 \mathrm{MHz}, \mathrm{DMSO}-d_{6}\right): \delta=3.36\left(6 \mathrm{H}, 2 \times \mathrm{CH}_{3}\right), 7.55,8.06,8.44$, and $8.73(2 \mathrm{H}, 2 \times \mathrm{CH}, \mathrm{BPY}) .{ }^{13} \mathrm{C}$ NMR (125.1 MHz, DMSO- $\left.d_{6}\right): \delta=40.29\left(\mathrm{CH}_{3}\right), 121.65$, $125.19,139.20$ and $148.50\left(2,2^{\prime}-\mathrm{BPY}\right), 189.87(\mathrm{NC}=\mathrm{S})$. Anal. calc. for $\mathrm{C}_{13} \mathrm{H}_{14} \mathrm{Cl}_{2} \mathrm{~N}_{3} \mathrm{~S}_{2} \mathrm{Au}$ (544.27): C, 28.69; H, 2.59; N, 7.72; S, 11.78\%. Found: C, 28.55; H, 2.51; N, 7.75; S, 11.80\%.

\subsection{Acute Toxicity Study (Single Dose)}

In the first step, an approximate $\mathrm{LD}_{50}$ was determined by the so called "staircase method", using a small number of animals (two for each dose), testing one dose of the compound-1 bipyridine gold (III) complex with dithiocarbamate ligand at a time (end point at $24 \mathrm{~h}$ ) and subsequently increasing the dose for the next group if needed. The arbitrarily administered doses of the bipyridine gold (III) complex with dithiocarbamate ligand were 250,500 , and $1000 \mathrm{mg} / \mathrm{kg}$. None of the animals died at $250 \mathrm{mg} / \mathrm{kg}$, one animal became very sick at $500 \mathrm{mg} / \mathrm{kg}$ after $20 \mathrm{~h}$ and had to be sacrificed, while both of the animals died at $1000 \mathrm{mg} / \mathrm{kg}$ : one at $18 \mathrm{~h}$ and the other at $22 \mathrm{~h}$. The dose was not increased further. The 
findings observed in the tissues of the animals used for dose selection are not included with the results of this study.

The five doses where there was the highest change of finding intraperitoneal $\mathrm{LD}_{50}$ were then chosen a. These were 50, 100, 200, 400, and $800 \mathrm{mg} / \mathrm{kg}$. These doses were then given to five groups of rats, with four animals in each group. A sixth group with four animals served as the control and was injected with distilled water. The animals were kept under continuous observation for the initial $2 \mathrm{~h}$ and were then sequentially observed every $2 \mathrm{~h}$ for any symptoms of toxicity to avoid the rodents experiencing any suffering. At the end of $24 \mathrm{~h}$, the number of rats that had died in each group were counted. The percentage of animals that had died at each dose level were transformed to probits [15], and $\mathrm{LD}_{50}$ was then determined according to the method of Miller and Tainter [16]. All of the living animals were sacrificed and dissected at the end of the $24 \mathrm{~h}$ period. The kidneys, livers as well as hearts of the animals were collected and preserved in $10 \%$ formalin for histological examination. The $\mathrm{LD}_{50}$ determined by the probits was $38.46 \mathrm{mg} / \mathrm{kg}$ body weight (Table 1 ).

Table 1. Determination of the lethal doses of gold complex for the determination of LD50 after intraperitoneal injection in rats $(n=24,4$ in each group).

\begin{tabular}{ccccc}
\hline Group & $\begin{array}{c}\text { Dose } \\
(\mathbf{m g} / \mathbf{k g})\end{array}$ & Log Dose & \% Dead & Probits \\
\hline 1 & 50 & 3.912 & 50 & 0.585 \\
\hline 2 & 100 & 4.605 & 100 & 0.783 \\
\hline 3 & 200 & 5.298 & 75 & 0.912 \\
\hline 4 & 400 & 5.991 & 100 & 0.973 \\
\hline 5 & 800 & 6.685 & 100 & 0.994 \\
\hline Control & 0 & 0 & 0 & 0 \\
\hline
\end{tabular}

\subsection{Subacute Toxicity Study}

A total of 20 rats of same age and approximately same weight (200-250 g) were used for this arm of the study, which continued for 42 days. Each rat was housed in a separate cage. The cages were kept at standardized temperature and humidity for one week before the start of intervention. They were given free access to food (rat-chow) and water. Food consumption was assessed daily by evaluating the leftover food, whereas the body weight of each rat was determined once before the commencement of treatment and weekly thereafter at a fixed time of the day.

Group-A (the control group, $n=6$ ) was injected with $1 \mathrm{~mL}$ of distilled water daily for 6 weeks by intraperitoneal injection. Group-B $(n=10)$ was injected $1 / 10$ of $L D_{50}$, a dose of $3.8 \mathrm{mg} / \mathrm{kg}$ body weight, daily for 6 weeks by intraperitoneal injection. Group-C $(n=4)$ was injected $1 / 5$ of $\mathrm{LD}_{50}$ for $7.6 \mathrm{mg} / \mathrm{kg}$ bodyweight daily for 6 weeks by intraperitoneal injection. The day of death was noted for the animals that died during the 6 weeks, and tissues were removed by dissection. At the end of 6 weeks, the alive animals were sacrificed. Tissues of all of the animals were put in $10 \%$ formaldehyde and were sent for histological evaluation (Table 2).

\subsection{Histopathological Evaluation}

An automated tissue processor (Tissue-tek VIP-5, from SAKURA) was employed for tissue processing. An initial two step fixation consisting of two cycles of two hours each for tissue immersion in buffered formalin was followed by washing the tissue in distilled water for half an hour for the removal of the fixative. The tissue was dehydrated by exposing it to an increasing concentration of alcohol, i.e., $70 \%, 90 \%$, and $100 \%$ for variable time periods, followed by clearing by the sequential immersion of the tissue in a mixture $50 \%$ xylene and alcohol and then pure xylene for half an hour each. The tissue was then impregnated in molten wax followed by embedding, formation of paraffin blocks, thin sectioning at 
4-5 um thickness, and, finally, hematoxylin and eosin (H\&E) staining. The prepared slides were then evaluated for histopathological alterations [17].

Table 2. Disposal of animals in subacute study $(n=20)$.

\begin{tabular}{|c|c|c|}
\hline Animal No. & Treatment & Fate (Upto 42 Days) \\
\hline A1-A6 & Distilled water & All survived, sacrificed on day 42 , tissues preserved for histopathology \\
\hline B3 & \multirow{3}{*}{$\begin{array}{l}3.8 \mathrm{mg} / \mathrm{kg} \mathrm{BW} \\
\left(1 / 10 \text { of } \mathrm{LD}_{50}\right)\end{array}$} & Died on day 22, tissues preserved for histopathology \\
\hline B9 & & Died on day 32, tissues preserved for histopathology \\
\hline B1, B2, B4-B8, B10 & & Survived up to day 42 , sacrificed, tissues preserved for histopathology \\
\hline $\mathrm{C} 1$ & \multirow{4}{*}{$\begin{array}{c}7.6 \mathrm{mg} / \mathrm{kg} \mathrm{BW} \\
\left(1 / 5 \text { of } \mathrm{LD}_{50}\right)\end{array}$} & Sacrificed on day 37 due to animal distress, tissues preserved for histopathology \\
\hline $\mathrm{C} 2$ & & Sacrificed on day 37 due to animal distress, tissues preserved for histopathology \\
\hline $\mathrm{C} 3$ & & Died on day 35, tissues preserved for histopathology \\
\hline $\mathrm{C} 4$ & & Died on day 36, tissues preserved for histopathology \\
\hline
\end{tabular}

H\&E-stained sections of renal, hepatic, and cardiac tissue from the rats administered the gold III compound and the control rats were examined for tubulopathies including necrosis, apoptosis, and tubular vacuolization. Additional findings of inflammation and vascular changes such as congestion were also noted in the renal tissue. Renal tubular necrosis was graded as follows: [18]

Grade $0=$ unremarkable renal tubules.

Grade 1 = renal tubular epithelial cell degeneration without significant apoptosis or necrosis.

Grades 2-5 = renal tubular epithelial cell degeneration including apoptosis and necrosis comprising $<25 \%,<50 \%,<75 \%$, and $\geq 75 \%$ of the renal tubules.

The hepatic tissue was evaluated for alterations in the architecture, portal triads, hepatocytes, sinusoids, inflammation, and the presence of degeneration, necrosis, and fatty change. These alterations were grouped into one of the following categories: [19]

Hepatitis: Acute, chronic, cholestatic, granulomatous.

Steatosis, steatohepatitis: microvesicular or macrovesicular.

Cholestasis: Bland or chlolestatic hepatitis.

Vascular Abnormalities: sinusoidal obstruction syndrome.

The cardiac tissue was analyzed for cytoplasmic vacuolization, variation in cardiac muscle fiber size, the hyalinization of muscle cells, fibrosis, muscle necrosis with contraction band formation, altered staining properties, and myocytolysis. Interstitial inflammation and interstitial edema were also noted. The extent of the changes was semi-quantitatively graded as follows: $[20,21]$

Grade 0: Unremarkable histological findings ( $0 \%)$.

Grade 1: Mild change involving up to a $30 \%$; initiation of change.

Grade 2: Moderate change involving 31-60\%.

Grade 3: Severe change involving 61-100\%; widespread changes identified.

\subsection{Analysis}

The results are summarized as frequencies arranged as crosstabs and were compared for significance using the chi square test in SPSS-IBM version 21.

\section{Results}

\subsection{Acute Toxicity Study}

In acute toxicity study in Group 1, with a dose of $50 \mathrm{mg} / \mathrm{kg}$, two out of four animals died before being euthanized for autopsy. In Group 2, 4, and 5, with dosages of 100, 400 , and $800 \mathrm{mg} / \mathrm{kg}$, respectively, all of the animals died before being euthanized, and Group 3, with a dose of $200 \mathrm{mg} / \mathrm{kg}$, in which three animals had died. Additionally, all 
the animals in these respective groups showed signs of increased motor activity, either before death or before being euthanized. Group 6 was the control group, which all of the animals remained alive. The following tables and figures show the renal (Tables 3 and 4 , Figures 3 and 4), hepatic (Tables 5 and 6, Figures 5 and 6), and cardiac (Table 7 and Figure 6) microscopic findings.

\subsection{Sub-Acute Toxicity Study}

This phase was divided into three groups. The first, Group A, which had six animals, was the control group. The renal, hepatic, and cardiac tissue were all unremarkable, as shown in Table 8 and Figure 7.

The second group, " $\mathrm{B}$ ". had 10 animals who were given a dosage of $3.8 \mathrm{mg} / \mathbf{k g}(\mathbf{1} / \mathbf{1 0}$ of LD50) body weight (dosage planned for 6 weeks). However, two of the animals, B1 and B2, died on day 22 and 32, respectively. (Table 8).

The third group, "C", had four animals who were given a dosage of $7.6 \mathrm{mg} / \mathbf{k g}(\mathbf{1} / 5$ of LD50) body weight (dosage planned for 6 weeks). However, C1 and C2 were sacrificed on day 37 , and $\mathrm{C} 3$ and $\mathrm{C} 4$ died on day 35 and 36, respectively (Table 8).

None of the animals in Group B or C had any signs of acute toxicity. However, two animals, $\mathrm{C} 1$ and $\mathrm{C} 2$, were sacrificed when signs of distress started to appear.

The histological findings are presented in Table 8 and Figure 8.
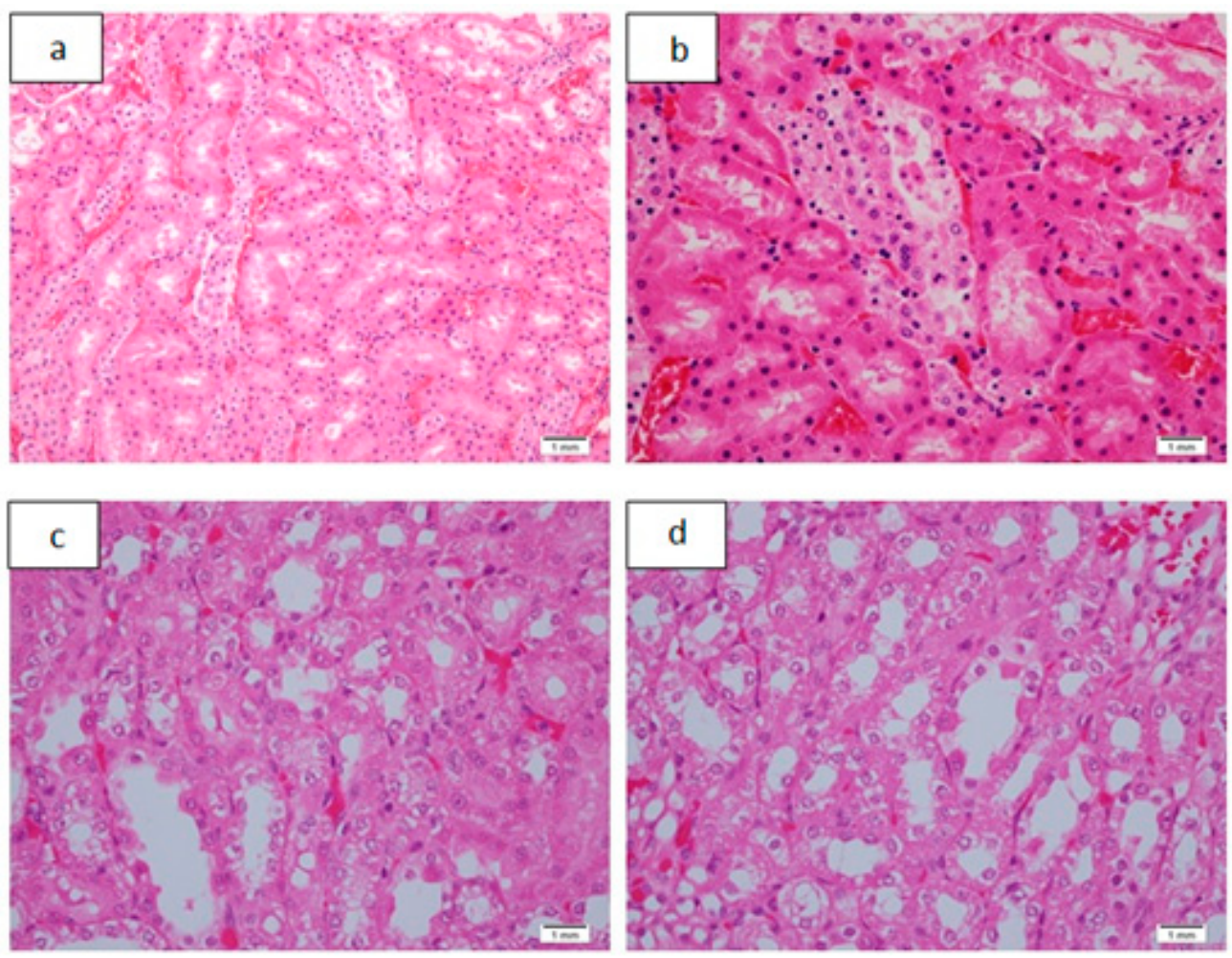

Figure 3. Renal tubular histological alterations as seen in acute toxicity study. (a): Renal tubular necrosis grade $1, \mathrm{H} \& \mathrm{E}, \times 20$. (b): Renal tubular necrosis grade 1 . Individual cell apoptosis can also be seen. $H \& E, \times 40$. (c): Renal tubular cytoplasmic vacuolization $H \& E, \times 40$. (d): Renal tubular cytoplasmic vacuolization with an occasional individual cell apoptosis, $\mathrm{H} \& \mathrm{E}, \times 40$. 

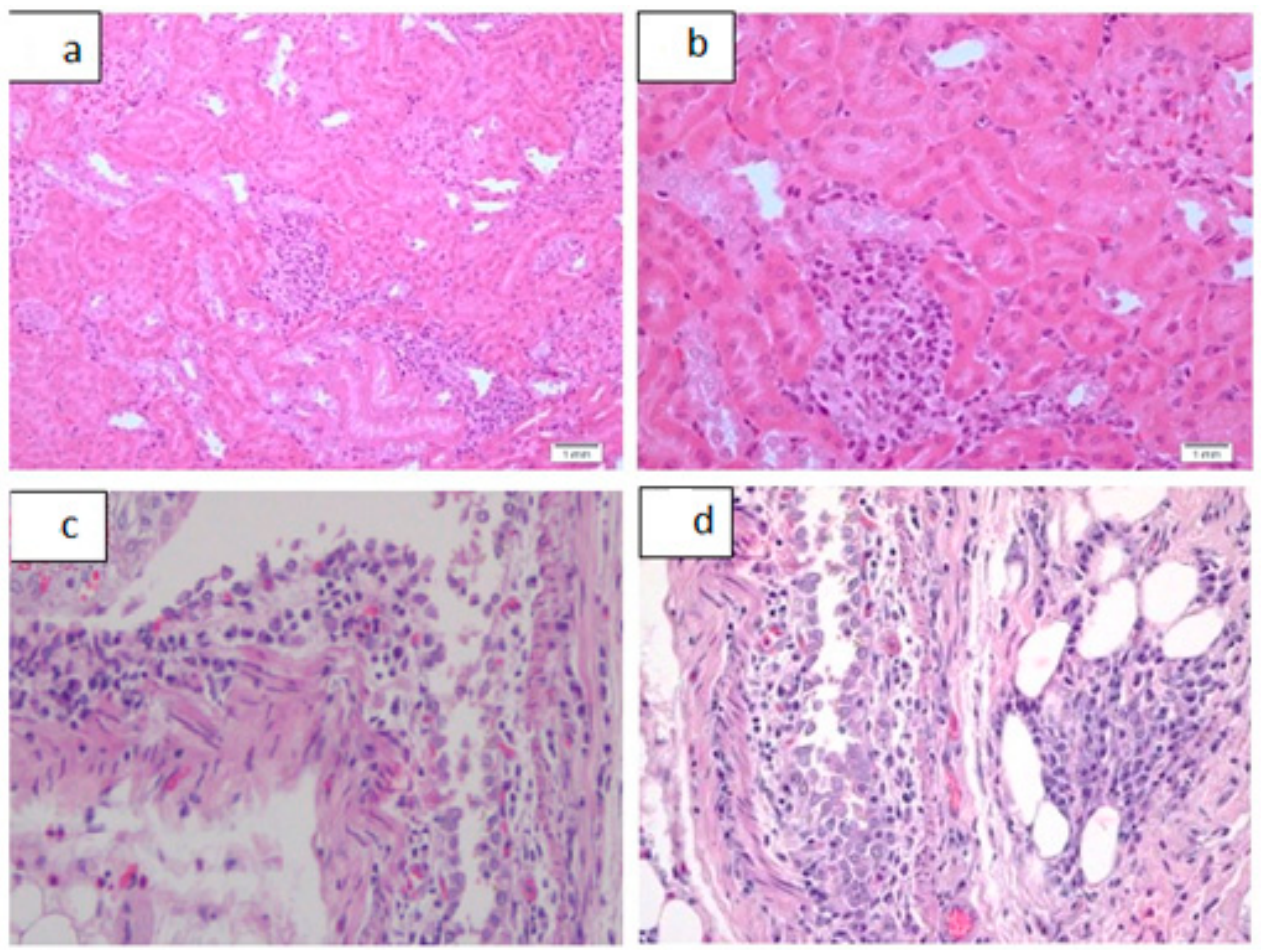

Figure 4. Spectrum of renal inflammatory changes as seen in acute toxicity study. (a): Moderate renal interstitial inflammation, $\mathrm{H} \& \mathrm{E}, \times 20$. (b): Moderate renal interstitial inflammation, $\mathrm{H} \& \mathrm{E}, \times 40$. (c): moderate plasmacytic pyelitis, $\mathrm{H} \& \mathrm{E}, \times 20$. (d): moderate plasmacytic pyelitis, $\mathrm{H} \& \mathrm{E}, \times 40$.
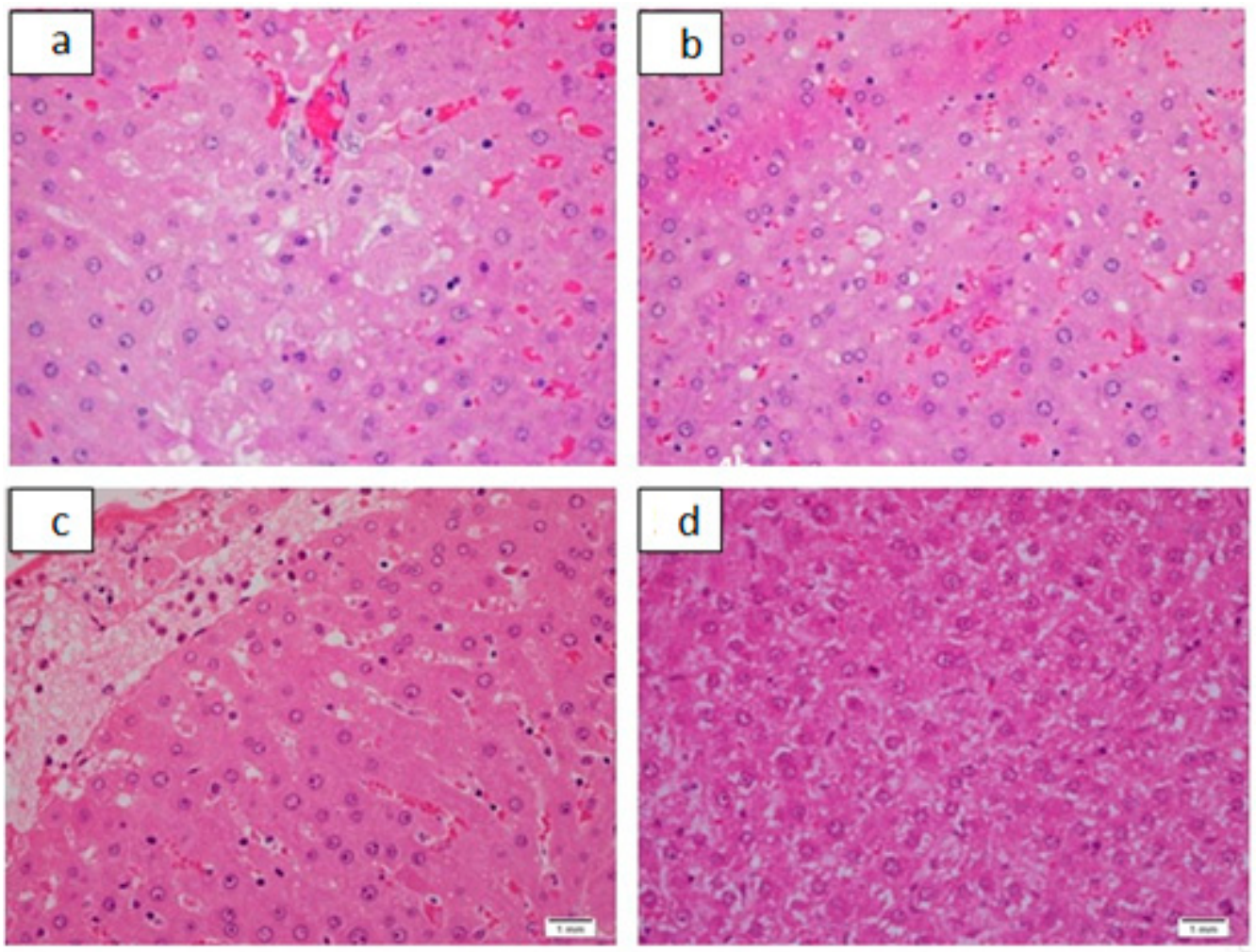

Figure 5. Hepatic histological alterations as seen in acute toxicity study. (a): Mixed hepatic micro and macrovesicular steatosis. An occasional apoptotic hepatocyte is also seen, $\mathrm{H} \& \mathrm{E}, \times 40$. (b): Mixed hepatic micro and macrovesicular steatosis, H\&E, $\times 40$. (c): Mild acute capsular inflammation, H\&E, $\times 40$. (d): Mild cytoplasmic vacuolization, $H \& E, \times 40$. 

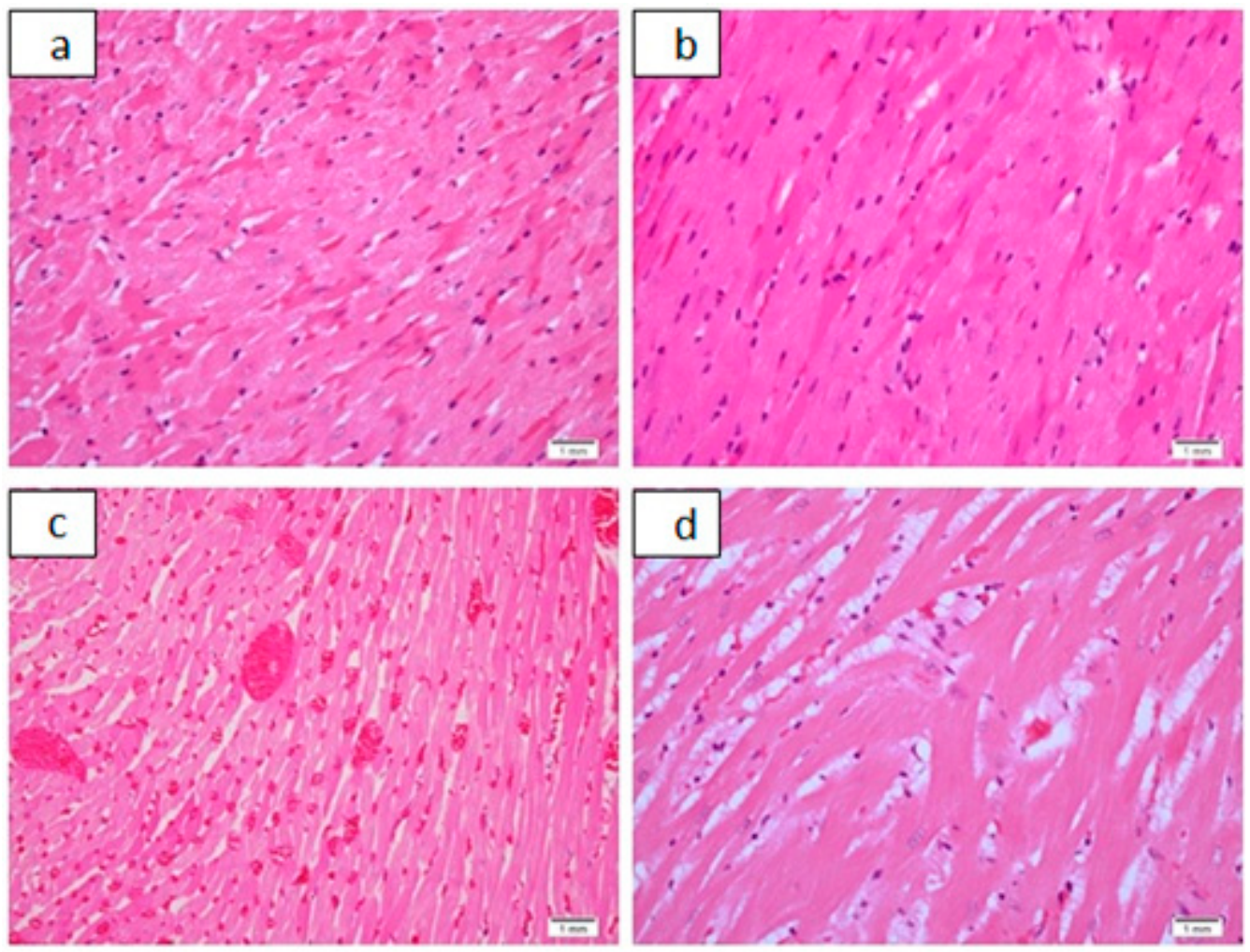

Figure 6. Cardiac histological findings as seen in acute toxicity study. (a): Moderate cardiac muscle fiber hyalinization, H\&E, $\times 20$. (b): Moderate cardiac muscle fiber hyalinization, $H \& E, \times 40$. (c): Marked cardiac congestion, H\&E, $\times 20$. (d): Mild cardiac cytoplasmic vacuolization, $H \& E, \times 40$.
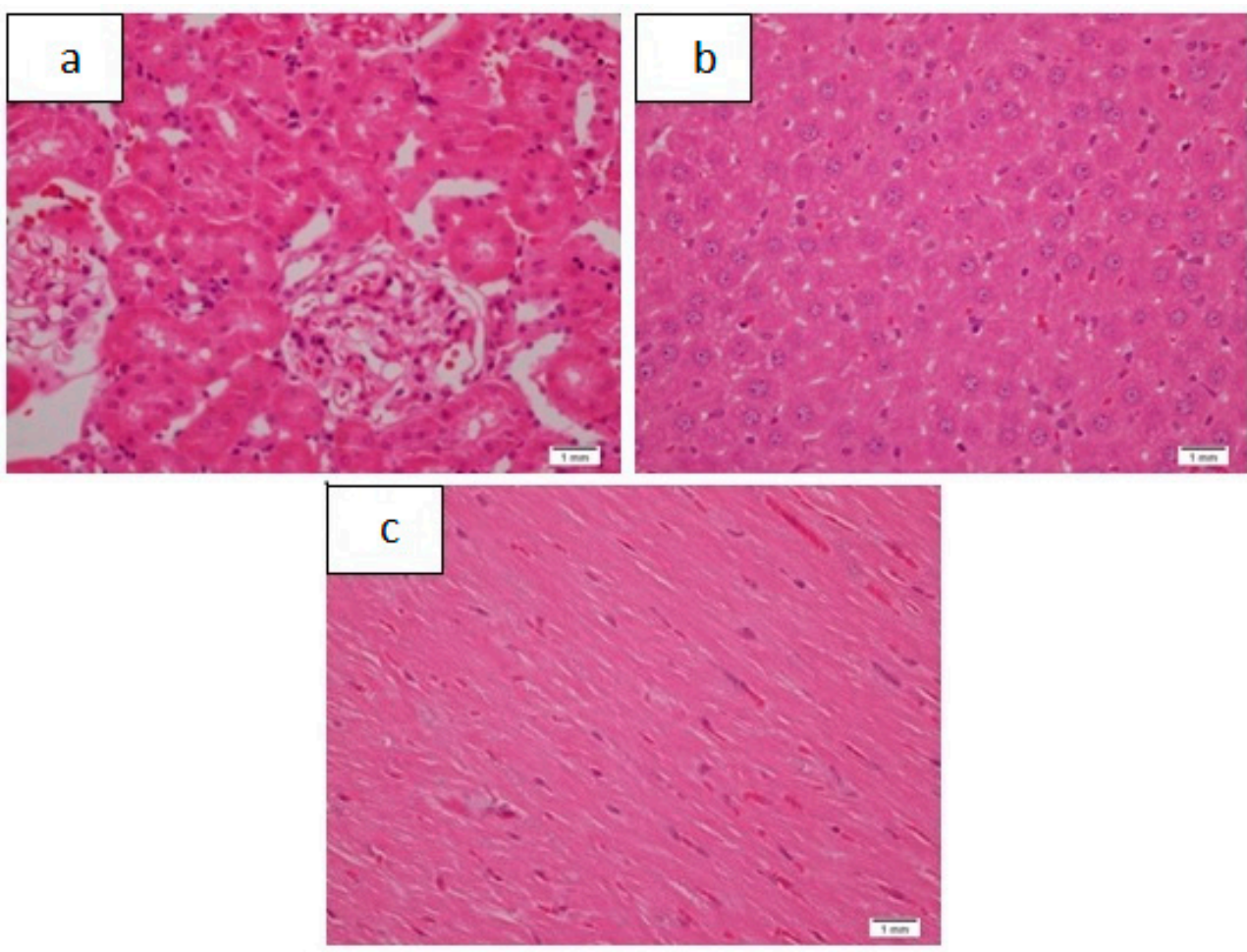

Figure 7. Unremarkable renal (a), hepatic (b), and cardiac (c) tissue, as seen in controls of sub-acute toxicity, H\&E, and $\times 20$. 
Table 3. Renal tubular necrosis and vacuolization in acute toxicity study. ( $n=24)$.

\begin{tabular}{|c|c|c|c|c|c|c|c|c|c|c|}
\hline \multirow{2}{*}{$\begin{array}{c}\text { Groups } \\
(n=4 \text { in } \\
\text { Each Group })\end{array}$} & \multirow{2}{*}{$\begin{array}{l}\text { Dose } \\
\mathrm{mg} / \mathrm{kg}\end{array}$} & \multicolumn{6}{|c|}{ Renal Tubular Necrosis Grade } & \multicolumn{3}{|c|}{ Renal Tubular Vacuolization } \\
\hline & & 5 & 4 & 3 & 2 & 1 & 0 & None & Less Than $\mathbf{5 0} \%$ & More Than $50 \%$ \\
\hline 1 & 50 & 0 & 0 & 0 & 0 & $\begin{array}{c}25 \% \\
(n=1) \\
\text { Figure 3a,b }\end{array}$ & $\begin{array}{l}75 \% \# \\
(n=3)\end{array}$ & $\begin{array}{l}100 \%{ }^{\#} \\
(n=4)\end{array}$ & 0 & 0 \\
\hline 2 & 100 & 0 & 0 & 0 & 0 & $\begin{array}{c}50 \% \\
(n=2)\end{array}$ & $\begin{array}{c}50 \% \\
(n=2)\end{array}$ & $\begin{array}{l}75 \% \# \\
(n=3)\end{array}$ & $\begin{array}{c}25 \% \\
(n=1) \\
\text { Figure 3c,d }\end{array}$ & 0 \\
\hline 3 & 200 & 0 & 0 & 0 & 0 & $\begin{array}{c}25 \% \\
(n=1)\end{array}$ & $\begin{array}{l}75 \% \# \\
(n=3)\end{array}$ & $\begin{array}{c}50 \% \\
(n=2)\end{array}$ & $\begin{array}{c}50 \% \\
(n=2)\end{array}$ & 0 \\
\hline 4 & 400 & 0 & 0 & 0 & 0 & $\begin{array}{c}50 \% \\
(n=2)\end{array}$ & $\begin{array}{c}50 \% \\
(n=2)\end{array}$ & $\begin{array}{c}25 \% \\
(n=1)\end{array}$ & $\begin{array}{l}75 \% \# \\
(n=3)\end{array}$ & 0 \\
\hline 5 & 800 & 0 & 0 & 0 & 0 & $\begin{array}{c}25 \% \\
(n=1))\end{array}$ & $\begin{array}{l}75 \% \# \\
(n=3)\end{array}$ & $\begin{array}{c}25 \% \\
(n=1)\end{array}$ & $\begin{array}{l}75 \% \# \\
(n=3)\end{array}$ & 0 \\
\hline $\begin{array}{c}6 \\
\text { (control) }\end{array}$ & - & 0 & 0 & 0 & 0 & 0 & 0 & 0 & 0 & 0 \\
\hline
\end{tabular}

Table 4. Pyelitis/interstitial inflammation and congestion in renal tissue in acute toxicity study $(n=24)$.

\begin{tabular}{|c|c|c|c|c|c|c|c|}
\hline \multirow{2}{*}{$\begin{array}{c}\text { Groups } \\
(n=4 \text { in } \\
\text { Each Group })\end{array}$} & \multirow{2}{*}{$\begin{array}{l}\text { Dose } \\
\mathrm{mg} / \mathrm{kg}\end{array}$} & \multicolumn{3}{|c|}{ Pyelitis/Interstitial Inflammation \% } & \multicolumn{3}{|c|}{ Congestion \% } \\
\hline & & None & Mild & $\begin{array}{c}\text { Mod/ } \\
\text { Marked }\end{array}$ & None & Mild & $\begin{array}{c}\text { Mod/ } \\
\text { Marked }\end{array}$ \\
\hline 1 & 50 & $\begin{array}{l}75 \%{ }^{\#} \\
(n=3)\end{array}$ & $\begin{array}{c}25 \% \\
(n=1)\end{array}$ & 0 & 0 & $\begin{array}{c}50 \% \\
(n=2)\end{array}$ & $\begin{array}{c}50 \% \\
(n=2)\end{array}$ \\
\hline 2 & 100 & $\begin{array}{l}75 \%{ }^{\#} \\
(n=3)\end{array}$ & $\begin{array}{c}25 \% \\
(n=1)\end{array}$ & 0 & 0 & 0 & $\begin{array}{l}100 \% \# \\
(n=4)\end{array}$ \\
\hline 3 & 200 & 0 & $\begin{array}{l}75 \% \# \\
(n=3)\end{array}$ & $\begin{array}{c}25 \% \\
(n=1) \\
\text { Figure } 4 \mathbf{a}-\mathbf{d}\end{array}$ & 0 & $\begin{array}{c}25 \% \\
(n=1)\end{array}$ & $\begin{array}{l}75 \%{ }^{\#} \\
(n=3)\end{array}$ \\
\hline 4 & 400 & $\begin{array}{c}25 \% \\
(n=1)\end{array}$ & $\begin{array}{c}25 \% \\
(n=1)\end{array}$ & $\begin{array}{c}50 \% \\
(n=2)\end{array}$ & 0 & 0 & $\begin{array}{l}100 \% \# \\
(n=4)\end{array}$ \\
\hline 5 & 800 & 0 & $\begin{array}{c}25 \% \\
(n=1)\end{array}$ & $\begin{array}{l}75 \% \# \\
(n=3)\end{array}$ & 0 & $\begin{array}{c}25 \% \\
(n=1)\end{array}$ & $\begin{array}{l}75 \% \# \\
(n=3)\end{array}$ \\
\hline $\begin{array}{c}6 \\
\text { (control) }\end{array}$ & - & 0 & 0 & 0 & 0 & 0 & 0 \\
\hline
\end{tabular}

Table 5. Extent of hepatic steatosis, necrosis, and congestion in acute toxicity study $(n=24)$.

\begin{tabular}{|c|c|c|c|c|c|c|c|c|c|c|}
\hline \multirow{2}{*}{$\begin{array}{c}\text { Groups } \\
(n=4 \text { in } \\
\text { Each Group }\end{array}$} & \multirow{2}{*}{$\begin{array}{c}\text { Dose } \\
\mathrm{mg} / \mathrm{kg}\end{array}$} & \multicolumn{3}{|c|}{ Steatosis } & \multicolumn{3}{|c|}{ Hepatocellular Necrosis/Degeneration } & \multicolumn{3}{|c|}{ Congestion } \\
\hline & & None & Mild & $\begin{array}{l}\text { Moderate } \\
\text { Marked }\end{array}$ & None & $\begin{array}{l}\text { Individual Cell } \\
\text { Degeneration }\end{array}$ & $\begin{array}{l}\text { Necrosis with } \\
\text { Inflammation }\end{array}$ & None & Mild & $\begin{array}{c}\text { Mod/ } \\
\text { Marked }\end{array}$ \\
\hline 1 & 50 & $\begin{array}{l}75 \% \# \\
(n=3)\end{array}$ & $\begin{array}{c}25 \% \\
(n=1) \\
\text { Figure } 5 \mathbf{a}, \mathbf{b}\end{array}$ & 0 & $\begin{array}{l}100 \% \\
(n=4)\end{array}$ & 0 & 0 & 0 & $\begin{array}{l}75 \% \# \\
(n=3)\end{array}$ & $\begin{array}{c}25 \% \\
(n=1)\end{array}$ \\
\hline 2 & 100 & $\begin{array}{l}75 \% \# \\
(n=3)\end{array}$ & $\begin{array}{c}25 \% \\
(n=1)\end{array}$ & 0 & $\begin{array}{l}75 \% \# \\
(n=3)\end{array}$ & $\begin{array}{c}25 \% \\
(n=1)\end{array}$ & 0 & $\begin{array}{c}25 \% \\
(n=1)\end{array}$ & $\begin{array}{c}75 \% \# \\
(n=3))\end{array}$ & 0 \\
\hline 3 & 200 & $\begin{array}{l}75 \% \# \\
(n=3)\end{array}$ & $\begin{array}{c}25 \% \\
(n=1)\end{array}$ & 0 & $\begin{array}{l}100 \% \# \\
(n=4)\end{array}$ & 0 & 0 & 0 & $\begin{array}{c}50 \% \\
(n=2)\end{array}$ & $\begin{array}{c}50 \% \\
(n=2)\end{array}$ \\
\hline 4 & 400 & $\begin{array}{l}75 \% \# \\
(n=3)\end{array}$ & $\begin{array}{c}25 \% \\
(n=1)\end{array}$ & 0 & $\begin{array}{c}25 \% \\
(n=1)\end{array}$ & $\begin{array}{l}75 \% \# \\
(n=3)\end{array}$ & 0 & 0 & 0 & $\begin{array}{l}100 \% \# \\
(n=4)\end{array}$ \\
\hline 5 & 800 & 0 & $\begin{array}{l}100 \% \# \\
(n=4)\end{array}$ & 0 & 0 & $\begin{array}{l}100 \% \# \\
(n=4)\end{array}$ & 0 & 0 & $\begin{array}{c}25 \% \\
(n=1)\end{array}$ & $\begin{array}{c}75^{\#} \\
(n=3)\end{array}$ \\
\hline 6 (control) & - & $\begin{array}{l}100 \% \# \\
(n=4)\end{array}$ & 0 & 0 & $\begin{array}{l}100 \% \# \\
(n=4)\end{array}$ & 0 & 0 & $\begin{array}{c}25 \% \\
(n=1)\end{array}$ & $\begin{array}{c}75^{\#} \\
(n=3)\end{array}$ & 0 \\
\hline
\end{tabular}

\#: $p$ Value $<0.05$ on $\mathrm{X}^{2}$ test. 
Table 6. Extent of portal, lobular, and capsular inflammation and cytoplasmic vacuolization in hepatic tissue in acute toxicity study $(n=24)$.

\begin{tabular}{|c|c|c|c|c|c|c|c|c|c|c|}
\hline \multirow{2}{*}{$\begin{array}{c}\text { Groups } \\
(n=4 \text { in } \\
\text { Each Group })\end{array}$} & \multirow{2}{*}{$\begin{array}{c}\text { Dose } \\
\mathrm{mg} / \mathrm{kg}\end{array}$} & \multicolumn{3}{|c|}{ Inflammation Portal/Lobular } & \multicolumn{3}{|c|}{ Capsular Acute Inflammation } & \multicolumn{3}{|c|}{ Cytoplasmic Vacuolization } \\
\hline & & None & Mild & $\begin{array}{l}\text { Moderate/ } \\
\text { Marked }\end{array}$ & None & Mild & $\begin{array}{c}\text { Mod/ } \\
\text { Marked }\end{array}$ & None & Mild & $\begin{array}{c}\text { Moderate/ } \\
\text { Marked }\end{array}$ \\
\hline 1 & 50 & $\begin{array}{l}75 \% \# \\
(n=3)\end{array}$ & $\begin{array}{c}25 \% \\
(n=1)\end{array}$ & 0 & $\begin{array}{c}25 \% \\
(n=1)\end{array}$ & $\begin{array}{c}75 \% \# \\
(n=3) \\
\text { Figure 5c }\end{array}$ & 0 & $\begin{array}{c}25 \% \\
(n=1)\end{array}$ & $\begin{array}{c}\begin{array}{c}75 \% \\
(n=3)\end{array} \\
\text { Figure 5d }\end{array}$ & 0 \\
\hline 2 & 100 & $\begin{array}{c}50 \% \\
(n=2)\end{array}$ & $\begin{array}{c}50 \% \\
(n=2)\end{array}$ & 0 & $\begin{array}{c}50 \% \\
(n=2)\end{array}$ & $\begin{array}{c}50 \% \\
(n=2)\end{array}$ & 0 & $\begin{array}{c}25 \% \\
(n=1)\end{array}$ & $\begin{array}{l}75 \% \\
(n=3)\end{array}$ & 0 \\
\hline 3 & 200 & $\begin{array}{l}75 \%{ }^{\#} \\
(n=3)\end{array}$ & $\begin{array}{c}25 \% \\
(n=1)\end{array}$ & 0 & $\begin{array}{c}50 \% \\
(n=2)\end{array}$ & $\begin{array}{c}25 \% \\
(n=1)\end{array}$ & $\begin{array}{c}25 \% \\
(n=1)\end{array}$ & 0 & $\begin{array}{c}50 \% \\
(n=2)\end{array}$ & $\begin{array}{c}50 \% \\
(n=2)\end{array}$ \\
\hline 4 & 400 & $\begin{array}{c}25 \% \\
(n=1)\end{array}$ & $\begin{array}{l}75 \%{ }^{\#} \\
(n=3)\end{array}$ & 0 & 0 & $\begin{array}{l}100 \%{ }^{\#} \\
(n=4)\end{array}$ & 0 & 0 & $\begin{array}{l}75 \%{ }^{\#} \\
(n=3)\end{array}$ & $\begin{array}{c}25 \% \\
(n=1)\end{array}$ \\
\hline 5 & 800 & 0 & $\begin{array}{c}100 \% \\
(n=4)\end{array}$ & 0 & $\begin{array}{c}50 \% \\
(n=2)\end{array}$ & $\begin{array}{c}50 \% \\
(n=2)\end{array}$ & 0 & 0 & $\begin{array}{l}75 \%{ }^{\#} \\
(n=3)\end{array}$ & $\begin{array}{c}25 \% \\
(n=1)\end{array}$ \\
\hline 6 (control) & - & $\begin{array}{l}75 \%{ }^{\#} \\
(n=3)\end{array}$ & $\begin{array}{c}25 \% \\
(n=1)\end{array}$ & 0 & $\begin{array}{c}50 \% \\
(n=2)\end{array}$ & $\begin{array}{c}50 \% \\
(n=2)\end{array}$ & 0 & $\begin{array}{c}75 \% \\
(n=3)\end{array}$ & $\begin{array}{c}25 \% \\
(n=1)\end{array}$ & 0 \\
\hline
\end{tabular}

Table 7. Cardiac histological findings in acute toxicity study $(n=24)$.

\begin{tabular}{|c|c|c|c|c|c|c|c|c|c|c|c|c|}
\hline \multirow{2}{*}{$\begin{array}{c}\text { Groups } \\
(n=4 \text { in } \\
\text { Each Group) }\end{array}$} & \multirow{2}{*}{$\begin{array}{c}\text { Dose } \\
\mathrm{mg} / \mathrm{kg}\end{array}$} & \multicolumn{4}{|c|}{ Cytoplasmic Vacuolization } & \multicolumn{4}{|c|}{ Hyalinization } & \multicolumn{3}{|c|}{ Congestion } \\
\hline & & None & Mild & Mod & Marked & None & Mild & Mod & Marked & None & Mild & $\begin{array}{c}\text { Mod/ } \\
\text { marked }\end{array}$ \\
\hline 1 & 50 & $\begin{array}{l}75 \% \# \\
(n=3) \\
\end{array}$ & $\begin{array}{c}25 \% \\
(n=1)\end{array}$ & 0 & 0 & $\begin{array}{l}75 \% \# \\
(n=3) \\
\end{array}$ & $\begin{array}{c}25 \% \\
(n=1) \\
\end{array}$ & 0 & 0 & 0 & $\begin{array}{c}50 \% \\
(n=2)\end{array}$ & $\begin{array}{c}50 \% \\
(n=2)) \\
\end{array}$ \\
\hline 2 & 100 & $\begin{array}{c}25 \% \\
(n=1)\end{array}$ & $\begin{array}{l}75 \% \# \\
(n=3)\end{array}$ & 0 & 0 & $\begin{array}{c}25 \% \\
(n=1)\end{array}$ & $\begin{array}{l}75 \%{ }^{\#} \\
(n=3)\end{array}$ & $\begin{array}{c}25 \% \\
(n=1) \\
\text { Figure } 6 \mathbf{a}, \mathbf{b}\end{array}$ & 0 & 0 & 0 & $\begin{array}{l}100 \% \# \\
(n=4)\end{array}$ \\
\hline 3 & 200 & $\begin{array}{c}50 \% \\
(n=2)\end{array}$ & $\begin{array}{c}50 \% \\
(n=2))\end{array}$ & 0 & 0 & & $\begin{array}{l}75 \%{ }^{\#} \\
(n=3)\end{array}$ & 0 & 0 & 0 & $\begin{array}{c}50 \% \\
(n=2)\end{array}$ & $\begin{array}{c}50 \% \\
(n=2) \\
\text { Figure 6c }\end{array}$ \\
\hline 4 & 400 & $\begin{array}{l}75 \% \# \\
(n=3)\end{array}$ & $\begin{array}{c}25 \% \\
(n=1)\end{array}$ & 0 & 0 & $\begin{array}{l}75 \% \# \\
(n=3)\end{array}$ & $\begin{array}{c}25 \% \\
(n=1)\end{array}$ & $\begin{array}{c}75 \\
(n=3)\end{array}$ & 0 & 0 & 0 & $\begin{array}{l}100 \%{ }^{\#} \\
(n=4)\end{array}$ \\
\hline 5 & 800 & $\begin{array}{c}50 \% \\
(n=2)\end{array}$ & $\begin{array}{c}50 \% \\
\text { Figure } 6 \mathrm{~d}\end{array}$ & 0 & 0 & & $\begin{array}{l}100 \% \# \\
(n=4)\end{array}$ & 0 & 0 & 0 & $\begin{array}{c}25 \% \\
(n=1)\end{array}$ & $\begin{array}{l}75 \% \# \\
(n=3)\end{array}$ \\
\hline $\begin{array}{c}6 \\
\text { (control) }\end{array}$ & - & 0 & 0 & 0 & 0 & 0 & 0 & 0 & 0 & $\begin{array}{c}25 \% \\
(n=1)\end{array}$ & $\begin{array}{l}75 \% \# \\
(n=3)\end{array}$ & 0 \\
\hline
\end{tabular}


Table 8. Histological findings in sub-acute toxicity study with of the doses of $3.8 \mathrm{mg} / \mathrm{kg}$ and $7.6 \mathrm{mg} / \mathrm{kg}(n=20)$.

\begin{tabular}{|c|c|c|c|c|c|c|c|c|c|c|c|c|}
\hline \multirow{3}{*}{$\begin{array}{l}\text { Animal } \\
\text { Groups }\end{array}$} & \multirow{3}{*}{ Dose } & \multicolumn{4}{|c|}{ RENAL } & \multicolumn{5}{|c|}{ HEPATIC } & \multirow{3}{*}{\multicolumn{2}{|c|}{$\begin{array}{l}\text { CARDIAC } \\
\text { Mild Focal Cardiac Fiber } \\
\text { Hyalinization }<10 \% \text { of } \\
\text { Tissue Examined }\end{array}$}} \\
\hline & & \multirow{2}{*}{ Normal } & \multirow{2}{*}{ Necrosis } & \multicolumn{2}{|c|}{ Interstitial Inflammation } & \multirow{2}{*}{ Normal } & \multicolumn{2}{|c|}{$\begin{array}{l}\text { Portal/Lobular } \\
\text { Inflammation }\end{array}$} & \multirow{2}{*}{$\begin{array}{c}\text { Mild } \\
\text { Capsular } \\
\text { Inflammation } \\
\text { *** }\end{array}$} & \multirow{2}{*}{$\begin{array}{c}\text { Mild } \\
\text { Cytoplasmic } \\
\text { Vacuolization } * * *\end{array}$} & & \\
\hline & & & & Mild & Mod & & Mild & Mod & & & & \\
\hline $\begin{array}{l}\text { A Control } \\
(n=6)\end{array}$ & 0 & $\begin{array}{c}100 \% \# \\
(n=6) \\
\text { Figure } 7 \mathrm{a}\end{array}$ & 0 & 0 & 0 & 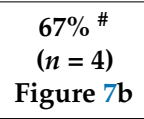 & $\begin{array}{c}33 \% \\
(n=2)\end{array}$ & 0 & $\begin{array}{c}16 \% \\
(n=1)\end{array}$ & 0 & $\begin{array}{c}100 \% \# \\
(n=6) \\
\text { Figure } 7 \mathrm{c}\end{array}$ & 0 \\
\hline $\begin{array}{c}\mathrm{B}^{*} \\
(n=10)\end{array}$ & $3.8 \mathrm{mg} / \mathrm{kg}$ & $\begin{array}{l}70 \% \# \\
(n=7)\end{array}$ & 0 & $\begin{array}{c}20 \% \\
(n=2) \\
\text { Figure } 8 \mathbf{a}, \mathbf{b}\end{array}$ & $\begin{array}{c}10 \% \\
(n=1)\end{array}$ & $\begin{array}{l}70 \%{ }^{\#} \\
(n=7)\end{array}$ & $\begin{array}{c}20 \% \\
(n=2)\end{array}$ & $\begin{array}{c}10 \% \\
(n=1)\end{array}$ & $\begin{array}{c}20 \% \\
(n=2)\end{array}$ & $\begin{array}{c}10 \% \\
(n=1) \\
\text { Figure 8c,d }\end{array}$ & $\begin{array}{l}80 \%{ }^{\#} \\
(n=8)\end{array}$ & $\begin{array}{c}20 \% \\
(n=2) \\
\text { Figure } 8 \mathbf{e}, \mathbf{f}\end{array}$ \\
\hline $\begin{array}{c}C^{* *} \\
(n=4)\end{array}$ & $\begin{array}{c}7.6 \\
\mathrm{mg} / \mathrm{kg}\end{array}$ & $\begin{array}{l}50 \%{ }^{\#} \\
(n=2)\end{array}$ & 0 & $\begin{array}{c}25 \% \\
(n=1)\end{array}$ & $\begin{array}{c}25 \% \\
(n=1)\end{array}$ & $\begin{array}{c}50 \% \\
(n=2)\end{array}$ & $\begin{array}{c}50 \% \\
(n=2)\end{array}$ & 0 & 0 & $\begin{array}{c}50 \% \\
(n=2)\end{array}$ & $\begin{array}{c}50 \% \\
(n=2)\end{array}$ & $\begin{array}{c}50 \% \\
(n=2)\end{array}$ \\
\hline
\end{tabular}

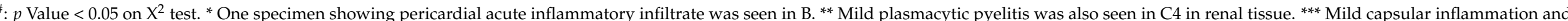

cytoplasmic vacuolization was seen in the same animals as those showing portal and lobular inflammation. Varying extents of congestion were seen in all groups, including the control group. 

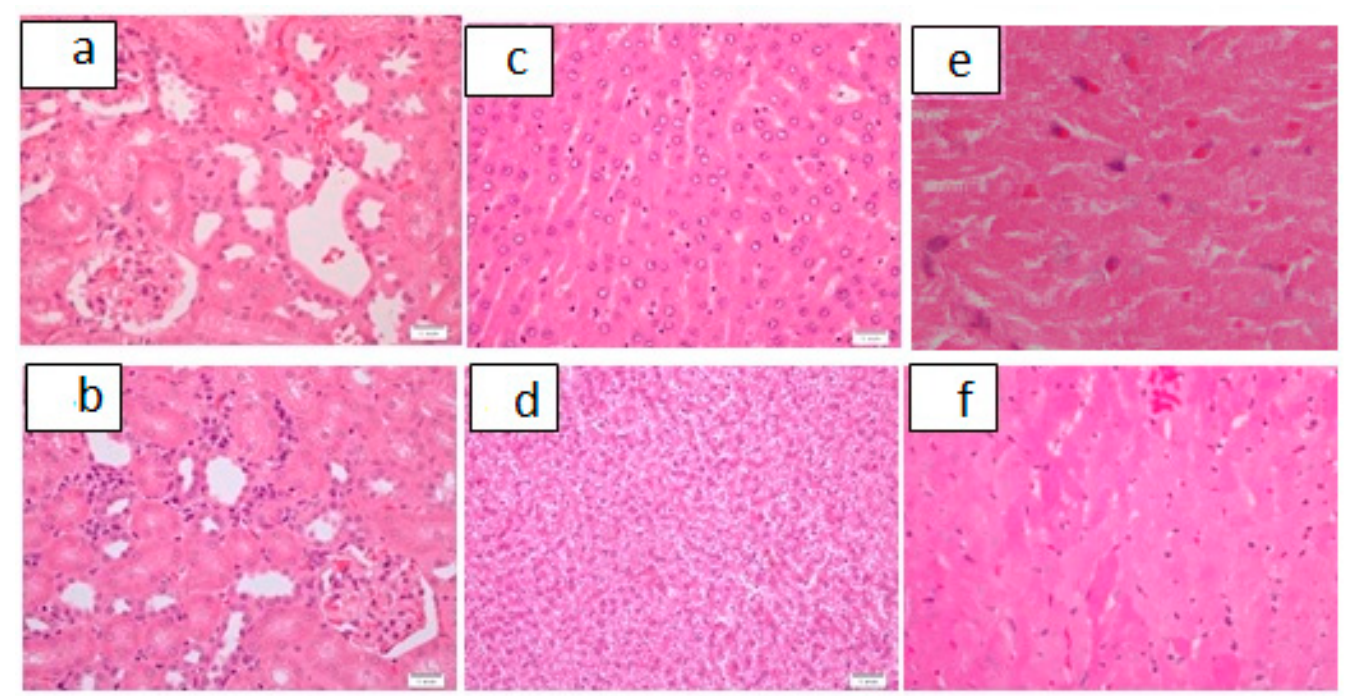

Figure 8. Histological picture of renal hepatic and cardiac tissue in subacute toxicity study at a dose of $3.8 \mathrm{mg} / \mathrm{kg}$. (a): Unremarkable renal tissue as seen in sub-acute toxicity $(3.8 \mathrm{mg} / \mathrm{kg})$, $\mathrm{H} \& \mathrm{E}, \times 40$. (b): Mild renal interstitial inflammation as seen in sub-acute toxicity $(3.8 \mathrm{mg} / \mathrm{kg})$, $\mathrm{H} \& \mathrm{E}, \times 40$. (c): Unremarkable hepatic tissue as seen in sub-acute toxicity $(3.8 \mathrm{mg} / \mathrm{kg}), \mathrm{H} \& \mathrm{E}, \times 40$. (d): Mild hepatic cytoplasmic vacuolization as seen in sub-acute toxicity $(3.8 \mathrm{mg} / \mathrm{kg}), \mathrm{H} \& \mathrm{E}, \times 40$. (e): Unremarkable cardiac tissue as seen in sub-acute toxicity $(3.8 \mathrm{mg} / \mathrm{kg}), \mathrm{H} \& \mathrm{E}, \times 40$. (f): Mild focal cardiac hyalinization and congestion as seen in sub-acute toxicity $(3.8 \mathrm{mg} / \mathrm{kg}), \mathrm{H} \& \mathrm{E}, \times 40$.

\section{Discussion}

Nephrotoxicity with concomitant renal failure is a major drawback in platinum-based anticancer drugs, especially cisplatin. This can present as acute tubular injury manifesting histologically as renal tubular cellular necrosis with inflammation. The process of necrosis can involve several necrosis inducing pathways that may include mitochondrial permeability transition-induced regulated necrosis, necroptosis, ferroptosis, or parthanatos [22]. Cisplatin, the major anti-cancerous agent in solid tumors, has nephrotoxity as its major initial and long-term drawback that can also lead to drug resistance. Its renal accumulation and concentration with reduced renal perfusion manifests morphologically as necrosis of the proximal tubular terminal portion and apoptosis, mainly the distal nephron [23]. In our bipyridine gold (III) complex with dicarbamato ligand, there was no evidence of renal tubular necrosis in either of the doses used in our subacute toxicity study (Figure 8 and Table 8).

In subacute toxicity with a dose of $3.8 \mathrm{mg} / \mathrm{kg}$ or $7.6 \mathrm{mg} / \mathrm{kg}$, the renal tubules remained unaffected, with no evidence of necrosis or renal tubular vacuolization. Mild to moderate congestion was seen. At a dose of $3.8 \mathrm{mg} / \mathrm{kg}$, mild to moderate focal interstitial inflammation was seen in three specimens (30\%). A dose of $7.6 \mathrm{mg} / \mathrm{kg}$ produced mild to moderate interstitial inflammation in two (50\%) of the study animals 1 (Figure 8 and Table 8). This finding suggests that the bipyridine gold (III) complex with dicarbamato ligand compound is safe renal wise.

As previously reported, the complex has proven its merit as a potent anticancer compound. This compound ligand was shown to have a low $\mathrm{IC}_{50}$ value, a high cellular uptake, and showed highly potent cytotoxicity against prostatic, ovarian, breast, lung, and Hodgkin lymphoma cells. It was proven to be 60-80-fold more active than cisplatin in inhibiting cellular proliferation in cisplatin resistant cells comprising a clone A2780cis of ovarian adenocarcinoma and s MCF-7 clone of breast cancer cells [13]. Therefore, a high anticancerous potency specifically against cisplatin resistant cancer stem lines and preserved renal tubules merits this complex to be investigated further in the context of new drug development. 
Oxiplatin, a third-generation platinum compound used in many malignancies, is less nephrotoxic compared to other platinum-based drugs. However, it has also been reported to induce renal tubular acidosis and/or necrosis. Oxaliplatin is a substrate for both the organic cation transporters (OCT2) present in proximal tubular basolateral membranes and the multidrug and toxin extrusion transport proteins (MATE, especially MATE2) present in proximal tubular brush border. An imbalance between OCT and MATE caused by platinum-based drugs lead to its diminished renal extrusion. Platinum compounds hence accumulate in renal tubules with concomitant renal damage [24]. It could be postulated that in the sub-acute toxicity study, the levels of MATE could be decreased by our bipyridine Gold (III) dicarbamato complex, which leads to its renal expulsion and hence preserved renal histology.

In the acute toxicity part of our study, however, grade 1 necrosis was seen. (Figure 3 and Table 3) The bipyridine gold (III) dicarbamato complex in the previously reported part of the study was proven to be a pro-apoptotic compound, and its anticancerous activity is exerted by inhibiting cellular proliferation by enhancing the mitochondrial intrinsic apoptotic pathway by a time-dependent activation of caspase 3 and 9, the promotion of mitochondrial permeability, and the release of cytochrome-c. All of these lead to the induction of apoptosis, evidenced by the fragmentation of the DNA analyzed by Apo-Direct analysis [13]. As such, the induction of apoptosis or grade 1 renal tubular necrosis seen in the acute toxicity part of the study could have been caused by the renal accumulation of the compound with this mechanism of action.

Renal tubular vacuolization was seen in the acute toxicity study (Figure 3 and Table 3 ). It has rarely been reported. Oxaplatin-induced renal tubular vacuolization was reported by Jobayari et al. [24]. It has also been reported after the administration of many types of drugs such as cisplatin, cyclophosphamide, immunoglobulins, sirolimus, imatinib, desferasirox, and cyclosporine [25], and hyperosmotic fluids, such as inulin, glucose, mannitol, sucrose, and radiocontrast agent, etc. [26]. Again, renal drug accumulation is considered to be the plausible mechanism. These findings and possible mechanisms imply that the bipyridine gold (III) dicarbamate complex that is under study accumulated in the renal tubules to cause minimal renal tubular necrosis and vacuolization. In sub-acute toxicity, however, the levels of the accumulated drug were so minimal that no renal damage occurred at histological levels (Figure 8 and Table 8). Such observations need to be validated by further investigations at the molecular level.

Another finding seen in the acute toxicity (Figure 4 and Table 4 ) and sub-acute toxicity parts (Figure 8 and Table 8 ) of the study is plasmacytic pyelitis and interstitial inflammation. Drugs can lead to an immunological reaction or a hapten-mediated disease, leading to renal damage. Drugs can act as an antigen with immune complex formation, leading to development of acute glomerulonephritis or acute interstitial nephritis. In the hapten mediated disease, part of a drug, any metabolite of the drug, or any substance modified by the drug can act as a hapten. These haptens can bind to the tubular epithelium or interstitial matrix, eliciting an immune reaction. This immune reaction can be an allergic reaction with involvement of IgE and eosinophils or it could be a cell-mediated immune reaction conducted by $\mathrm{CD} 4+\mathrm{T}$ lymphocytes [27]. These drug-related immune reactions are categorized as idiosyncratic or Type $\mathrm{B}$ reactions. These are unrelated to the mechanism of action of the drug [22]. The production of inflammasomes by the drug can also be considered as a possible mechanism that induces interstitial inflammation [28]. Cisplatin may also activate complex signaling pathways, including inflammatory pathways [29]. The extent of inflammation seen in sub-acute toxicity with our compound was predominantly mild and focal. This level of inflammatory changes needs to be evaluated clinically, and blood tests need to be conducted to assess the extent of renal impairment; however, in the absence of other findings of renal damage such as necrosis and tubular vacuolization, these seem to be of relatively less significance.

Regarding the hepatic tissue, in the sub-acute toxicity study at a dose of $3.8 \mathrm{mg} / \mathrm{kg}$ and $7.6 \mathrm{mg} / \mathrm{kg}$, the hepatic tissue only showed inflammatory changes comprising mild 
capsular acute inflammation, mild to moderate focal lobular and portal inflammation, and, occasionally, mild focal cytoplasmic vacuolization (Figure 8 and Table 8). In acute toxicity part of the study, the toxic effects were more apparent, with appearance of mild steatosis in all groups and a graded pattern of individual cellular degeneration/necrosis as well as increased cytoplasmic vacuolization along with inflammatory changes (Figure 5 and Table 5). Congestion was a finding that was seen to varying extents the in acute, sub-acute, and control groups.

The liver is the main organ involved in drug metabolism and clearance and is therefore the most susceptible to drug induced liver injury. This mode of hepatic injury is the main reason for the termination of drugs in clinical trials and the withdrawal of drugs used for treatment purposes. This may have a dose dependent or an idiosyncratic dose independent presentation [30].

Massive hepatotoxicity is seen with cisplatin, doxorubicin, and 5- Flurouracil (5FU), even at therapeutic doses. In a study by El-Sayyad et al., treatment at low doses of $0.2 \mathrm{mg} / \mathrm{kg}$ body weight of cisplatin and doxorubicin or $10 \mathrm{mg} / \mathrm{kg}$ of $5-\mathrm{FU}$ in rats for 20 days was shown to induce hepatic cellular vacuolar degeneration and apoptosis along with vascular alterations comprising endothelial cell damage with perivascular mononuclear cellular infiltration and periportal fibrosis. A dose of $1 \mathrm{mg} / \mathrm{kg}$ body weight, the therapeutic dose of cisplatin and doxorubicin, led to hepatocellular necrosis with dissolution of the hepatic cords with dense inflammatory cellular infiltration. An increased tendency for hepatic fibrosis and granulomatous lesions was observed with doxorubicin treatment. Cisplatin and doxorubicin showed more hepatic damage compared to the treatment with 5-FU [31].

Mild capsular inflammation, mild lobular and portal inflammation, and mild congestion are the findings that were seen in the sub-acute toxicity part of the study, (Figure 8 and Table 8); however, the same changes were observed in the controls (Figure 7 and Table 8). The presence of these findings in low-dose and drug-free animals implies that some other mechanism was responsible for these alterations. The drug-induced mechanism is not the only plausible mechanism that can cause these inflammatory changes.

Regarding steatosis, drug-induced steatosis or steatohepatitis is reported in less than $2 \%$ of cases of nonalcoholic steatohepatitis (NASH). Drugs that can induce NASH may show a definite metabolic alteration, such as tamoxifen, which involves the decreased incorporation of free fatty acids into very low-density lipoproteins along with their decreased hepatic expulsion or can be independent of any known definite mechanism, such as perhexiline maleate or amiodarone, or may produce sporadic cases of NASH, such as carbamazepine $[32,33]$. In the conversion of hepatocytes of acetyl-CoA to fatty acids is regulated by sterol regulatory element-binding protein- $1 \mathrm{c}$, a membrane-bound transcription factor that can activate fatty acid synthase, acetyl-CoA carboxylase, stearoyl-CoA desaturase, and ATP-citrate lyase, leading to lipogenesis. Lipid hemostasis is also modulated by another transcription factor, the peroxisome proliferator-activated receptors. The mitochondrial accumulation of drugs with resultant interference in mitochondrial electron transport chain and $\beta$-oxidation can also cause drug-induced liver injury and NASH $[34,35]$. The basic mechanism that underlies the development of steatosis in our compound has yet to be unraveled, but it can safely be stated that the lack of steatosis in the sub-acute toxicity study renders this compound as a hepatic-safe compound. However, such observations need to be validated with more extensive clinical and biochemical studies.

Hepatocytic individual cell degeneration/necrosis was seen in the acute toxicity study (Figure 5 and Table 5). Drug-induced hepatocytic necrosis may include massive, sub massive, and zonal necrosis. The combined pattern of necrosis and inflammation seen in drug-induced liver injury may include massive, sub massive, and zonal necrosis. Massive necrosis is characterized by pan-acinar necrosis with the complete loss of hepatocytes and parenchymal collapse between the residual portal areas. It has been shown to involve multiple contiguous acini. Ductular proliferation, varying extents of portal inflammation, and evidence of regeneration in the remaining hepatic parenchyma is also seen. Necrosis 
involving zone 3 is a feature of zonal necrosis. There is usually no associated portal or parenchymal inflammation. Zonal necrosis is seen to have some chemotherapeutic agents [36]. The necrosis seen with our compound, even in very high doses, was very minimal, and there was no necrosis observed in the sub-acute toxicity study, imparting the attribute of hepatic safety to this compound.

The cardiac findings in the sub-acute toxicity part (Figure 8 and Table 8) with a dosage of $3.8 \mathrm{mg} / \mathrm{kg}$ cardiac muscle fibers were unremarkable in $80 \%(n=8)$. Mild cardiac muscle fiber hyalinization was seen in $20 \%(n=2)$ of specimens in less than $10 \%$ of the material examined. Pericardial acute inflammatory cellular infiltrate was seen in $10 \%$ $(n=1)$. A dosage of $7.6 \mathrm{mg} / \mathrm{kg}$ led to mild focal hyalinized fibers in $50 \%$ of specimens $(n=2)$. In the acute toxicity study (Figure 6 and Table 7$)$, the cardiac histological alterations were mild cytoplasmic vacuolization in all specimens, mild to moderate hyalinization, and congestion. Congestion was a finding that was also seen in the controls and in the sub-acute toxicity study.

Cardiac hyalinization and fibrosis manifesting as cardiomyopathy is a side effect of many anti-cancerous agents. Doxorubicin, an effective anticancerous agent that is widely used in the treatment of solid tumors has an associated cardiomyopathy that has been seen histologically as areas of and scattered vacuolated cardiomyocytes and foci of patchy and widespread cardiac interstitial fibrosis. When the acute damage is healed, fibroblastic proliferation with histiocytic infiltration is seen in healed areas [37]. Doxorubicin kills cancerous cells by intercalating into DNA with topoisomerase II (TOP2) inhibition and caspase activation [38]. Its cardiotoxic effects are mainly manifested by the generation of reactive oxygen species [39]. New bipyridine gold (III) dithiocarbamate-containing complexes (the compound in this study) have been previously reported to overproduce mitochondrial reactive oxygen species [13]. Their cardiac hyalinization and vacuolization may be attributed to this mechanism. However, the damage observed in our cases was not that extensive, as only focal cytoplasmic vacuolization and hyalinized fibres were seen in the sub-acute toxicity study (Figure 8 and Table 8 ).

As far as cytoplasmic vacuolization is concerned, it has been reported to be reversible, transient, or irreversible. Vacuolar formation could just be an adaptation to a variety of environmental alterations [40]. Its transient form is mostly caused by weak basic aminecontaining lipophilic compounds that can pass through cell and organellar membranes, increasing intraorganellar osmotic pressure with water diffusion and the development of vacuolization. Irreversible vacuolization results due to cytotoxic stimulus with cytopathological damage culminate as cell death [41]. This pattern of vacuolization may be caused by caspase-independent cell death mechanisms comprising oncosis, methuosis, necroptosis, or paraptosis [42]. We had vacuolization in cardiac specimens in the acute and sub-acute toxicity parts of the study. Whether this was just an adaptive response or an initiation of cell death needs to be elaborated upon with more studies at molecular levels. The changes observed, however, seem to be adaptive and reversible, as no evidence of necrosis was discerned in cardiac tissue, even at high doses in the acute toxicity part of the study.

Drug resistance to chemotherapeutic agents is a central problem in oncology. The results of this investigation have identified a new class of gold (III) compounds as potential candidates for application in cancer chemotherapy. This is based on the demonstration that gold (III) compounds are effective in cisplatin-resistant cell lines. Moreover, since these compounds contain a dithiocarbamate group that is capable of preventing a reaction with other sulfur-containing proteins, we hypothesize a reduced toxicity in vivo. Thus, a gold (III) drug with an appropriate ligand, such as that presented in this study, has the potential to be an alternative to platinum-based drugs in cancer treatment.

Conclusion: In the subacute toxicity part of the study with a dose of $3.8 \mathrm{mg} / \mathrm{kg}$ or $7.6 \mathrm{mg} / \mathrm{kg}$, the renal tubules remained unaffected, with no evidence of necrosis or renal tubular vacuolization. The cardiac muscle fibers also remained unremarkable in $80 \%$ of the specimens, with mild focal muscle fiber hyalinization seen in the remaining $20 \%$. Mild focal renal and hepatic inflammatory changes were the only concomitant alterations. 
Such minimal histological alterations may attribute this bipyridine gold (III) complex with dithiocarbamates ligand to be safe in animal studies and strongly suggest it as a potential candidate for clinical trials.

Author Contributions: Conceptualization, A.A.I., D.M.A.-T. and M.A.; methodology, A.B., A.A., D.M.A.-T., A.A.I., M.A., and S.A.; software, A.A.I. and A.B.; validation, D.M.A.-T. and A.A.I.; formal analysis, A.A., A.A.I., and S.A.; investigation, A.B., A.A., and S.A.; resources, D.M.A.-T., A.A.I., A.B. and A.A.; data curation, A.B., A.A., A.A.I., and S.A.; writing-original draft preparation, A.B., A.A. and A.A.I.; writing-review and editing, D.M.A.-T., M.A. and S.A.; visualization, A.B., A.A.; supervision, D.M.A.-T., A.A.I.; project administration, D.M.A.-T., A.A.I.; funding acquisition, A.A.I. and D.M.A.-T.; All authors have read and agreed to the published version of the manuscript.

Funding: This project was funded by the Deanship of Scientific Research at King Fahd University of Petroleum \& Minerals for funding this work through project No. IN171005.

Institutional Review Board Statement: The study was conducted according to the guidelines of the Declaration of Helsinki, and approved by the ethical committee at Deanship of Scientific Research, King Fahd University of Petroleum \& Minerals (No: IN171005, dated 15 April 2018) and in continuation of KACST project No: 14-MED-64-04, dated 13 January 2015 (17/3/1436 H) at Imam Abdulrahman Bin Faisal University (formerly University of Dammam).

Informed Consent Statement: Not applicable.

Data Availability Statement: Not applicable.

Conflicts of Interest: There is no conflict of interests.

\section{References}

1. Kostova, I. Platinum complexes as anticancer agents. Recent Patent Anticancer. Drug Discov. 2006, 1, 1-22. [CrossRef] [PubMed]

2. Astolfi, L.; Ghiselli, S.; Guaran, V.; Chicca, M.; Simoni, E.; Olivetto, E.; Lelli, G.; Martini, A. Correlation of adverse effects of cisplatin administration in patients affected by solid tumours: A retrospective evaluation. Oncol. Rep. 2013, 29, 1285-1292. [CrossRef] [PubMed]

3. Casini, A.; Wai-Yin Sun, R.; Ott, I. Medicinal chemistry of gold anticancer metallodrugs (Chapter-7). In Metallo-Drugs: Development and Action of Anticancer Agents; Sigel, A., Sigel, H., Freisinger, E., Sigel, R., Eds.; De Gruyter: Berlin, Germany; Boston, FL, USA, 2018; pp. 199-218. [CrossRef]

4. Fernández-Moreira, V.; Herrera, R.P.; Gimeno, M.C. Anticancer properties of gold complexes with biologically relevant ligands. Pure Appl. Chem. 2019, 91, 247-269. [CrossRef]

5. Yang, Z.; Jiang, G.; Xu, Z.; Zhao, S.; Liu, W. Advances in alkynyl gold complexes for use as potential anticancer agents. Coord. Chem. Rev. 2020, 423, 213492. [CrossRef]

6. Olszewski, U.; Hamilton, G. A better platinum-based anticancer drug yet to come? Anticancer Agents Med. Chem. 2010, 10, 293-301. [CrossRef]

7. Janković, S.M.; Djeković, A.; Bugarčić, Z.; Janković, S.V.; Lukić, G.; Folić, M.; Canović, D. Effects of aurothiomalate and gold(III) complexes on spontaneous motility of isolated human oviduct. Biometals 2012, 25, 919-925. [CrossRef]

8. Kalayda, G.V.; Wagner, C.H.; Jaehde, U. Relevance of copper transporter 1 for cisplatin resistance in human ovarian carcinoma cells. J. Inorg. Biochem. 2012, 116, 1-10. [CrossRef] [PubMed]

9. Buckley, R.G.; Elsome, A.M.; Fricker, S.P.; Henderson, G.R.; Theobald, B.R.; Parish, R.V.; Howe, B.P.; Kelland, L.R. Antitumor properties of some 2-[(dimethylamino)methyl]phenylgold(III) complexes. J. Med. Chem. 1996, 39, 5208-5214. [CrossRef]

10. Calamai, P.; Carotti, S.; Guerri, A.; Mazzei, T.; Messori, L.; Mini, E.; Orioli, P.; Speroni, G.P. Cytotoxic effects of gold(III) complexes on established human tumor cell lines sensitive and resistant to cisplatin. Anticancer Drug Des. 1998, 13, 67-80.

11. Ahmed, A.; Al Tamimi, D.M.; Isab, A.A.; Alkhawajah, A.M.; Shawarby, M.A. Histological changes in kidney and liver of rats due to gold (III) compound [Au(en)Cl(2)]Cl. PLoS ONE 2012, 7, e51889.

12. Marzano, C.; Ronconi, L.; Chiara, F.; Giron, M.C.; Faustinelli, I.; Cristofori, P.; Trevisan, A.; Fregona, D. Gold (III)-dithiocarbamato anticancer agents: Activity, toxicology and histopathological studies in rodents. Int. J. Can. 2011, 129, 487-496. [CrossRef] [PubMed]

13. Altaf, M.; Monim-Ul-Mehboob, M.; Kawde, A.N.; Corona, G.; Larcher, R.; Ogasawara, M.; Casagrande, N.; Celegato, M.; Borghese, C.; Siddik, Z.H.; et al. New bipyridine gold(III) dithiocarbamate-containing complexes exerted a potent anticancer activity against cisplatin-resistant cancer cells independent of p53 status. Oncotarget 2017, 8, 490-505. [CrossRef] [PubMed]

14. OECD Series on Principles of Good Laboratory Practice (GLP) and Compliance Monitoring. Available online: http://www.oecd. $\mathrm{org} /$ chemicalsafety/testing/oecdseriesonprinciplesofgoodlaboratorypracticeglpandcompliancemonitoring.htm (accessed on 2 October 2019).

15. Ghosh, M.N. Satistical Analysis, Fundamentals of Experimental Pharmacology, 2nd ed.; Scientific Book Agency: Calcutta, India, $1984 ;$ p. 189. 
16. Miller, L.C.; Tainter, M.L. Estimation of ED50 and its error by means of log-probit graph paper. Proc. Soc. Exp. Biol. Med. 1944, 57, 261. [CrossRef]

17. Underwood, J.C.E. Histochemistry. Theoretical and Applied. Vol. 2: Analytical Technology Pearse AGE, 4th ed.; Churchill Livingstone: Edinburgh, UK, 1985.

18. Zhang, J.; Brown, R.P.; Shaw, M.; Vaidya, V.S.; Zhou, Y.; Espandiari, P.; Sadrieh, N.; Stratmeyer, M.; Keenan, J.; Kilty, C.G.; et al. Immunolocalization of Kim-1, RPA-1, and RPA-2 in Kidney of Gentamicin-, Mercury-, or Chromium-treated Rats: Relationship to Renal Distributions of iNOS and Nitrotyrosine. Toxicol. Pathol. 2008, 36, 397-409. [CrossRef] [PubMed]

19. Ramachandran, R.; Kakar, S. Histological patterns in drug-induced liver disease. J. Clin. Pathol. 2009, 62, 481-492. [CrossRef]

20. Lim, A.Y.; Segarra, I.; Chakravarthi, S.; Akram, S.; Judson, J.P. Histopathology and biochemistry analysis of the interaction between sunitinib and paracetamol in mice. BMC Pharmacol. 2010, 10, 14. [CrossRef]

21. Faqi, A.S. A Comprehensive Guide to Toxicology in Preclinical Drug Development; Academic Press: Cambridge, MA, USA, 2012.

22. Izzedine, H.; Perazella, M.A. Anticancer Drug-Induced Acute Kidney Injury. Kidney Int. Rep. 2017, 2, 504-514. [CrossRef]

23. Arany, I.; Safirstein, R.L. Cisplatin nephrotoxicity. Semin. Nephrol. 2003, 23, 460-464. [CrossRef]

24. Joybari, A.Y.; Sarbaz, S.; Azadeh, P.; Mirafsharieh, S.A.; Rahbari, A.; Farasatinasab, M.; Mokhtari, M. Oxaliplatin-induced renal tubular vacuolization. Ann. Pharmacother. 2014, 48, 796-800. [CrossRef]

25. Laftavi, M.R.; Weber-Shrikant, E.; Kohli, R.; Patel, S.; Feng, L.; Said, M.; Dayton, M.; Pankewycz, O. Sirolimus-induced isometric tubular vacuolization: A new sirolimus histopathologic manifestation. Transplant. Proc. 2010, 42, 2547-2550. [CrossRef]

26. Naesens, M.; Kuypers, D.R.J.; Sarwal, M. Calcineurin inhibitor nephrotoxicity. Clin. J. Am. Soc. Nephrol. 2009, 4, 481-508. [CrossRef] [PubMed]

27. Bartoli, E. Adverse effects of drugs on the kidney. Eur. J. Intern. Med. 2016, 28, 1-8. [CrossRef] [PubMed]

28. Cho, T.; Uetrecht, J. How Reactive Metabolites Induce an Immune Response That Sometimes Leads to an Idiosyncratic Drug Reaction. Chem. Res. Toxicol. 2017, 30, 295-314. [CrossRef] [PubMed]

29. Pabla, N.; Dong, Z. Cisplatin nephrotoxicity: Mechanisms and renoprotective strategies. Kidney Int. 2008, 73, 994-1007. [CrossRef]

30. Björnsson, E.S.; Bergmann, O.M.; Björnsson, H.K.; Kvaran, R.B.; Olafsson, S. Incidence, presentation, and outcomes in patients with drug-induced liver injury in the general population of Iceland. Gastroenterology 2013, 144, 1419-1425. [CrossRef]

31. El-Sayyad, H.I.; Ismail, M.F.; Shalaby, F.M.; Abou-El-Magd, R.F.; Gaur, R.L.; Fernando, A.; Raj, M.H.G.; Ouhtit, A. Histopathological effects of cisplatin, doxorubicin and 5-flurouracil (5-FU) on the liver of male albino rats. Int. J. Biol. Sci. 2009, 5, 466-473. [CrossRef]

32. Grieco, A.; Forgione, A.; Miele, L.; Vero, V.; Greco, A.V.; Gasbarrini, A.; Gasbarrini, G. Fatty liver and drugs. Eur. Rev. Med. Pharmacol. Sci. 2005, 9, 261-263.

33. Zhao, F.; Xie, P.; Jiang, J.; Zhang, L.; An, W.; Zhan, Y. The effect and mechanism of tamoxifen-induced hepatocyte steatosis in vitro. Int. J. Mol. Sci. 2014, 15, 4019-4030. [CrossRef]

34. Vitins, A.P.; Kienhuis, A.S.; Speksnijder, E.N.; Roodbergen, M.; Luijten, M.; Van der Ven, L.T.M. Mechanisms of amiodarone and valproic acid induced liver steatosis in mouse in vivo act as a template for other hepatotoxicity models. Arch. Toxicol. 2014, 88, 1573-1588. [CrossRef]

35. Rabinowich, L.; Shibolet, O. Drug Induced Steatohepatitis: An Uncommon Culprit of a Common Disease. Biomed. Res. Int. 2015, 2015, 168905. [CrossRef]

36. Kleiner, D.E. The histopathological evaluation of drug-induced liver injury. Histopathology 2017, 70, 81-93. [CrossRef]

37. Zhao, L.; Zhang, B. Doxorubicin induces cardiotoxicity through upregulation of death receptors mediated apoptosis in cardiomyocytes. Sci. Rep. 2017, 7, 44735. [CrossRef] [PubMed]

38. Zhang, S.; Liu, X.; Bawa-Khalfe, T.; Lu, L.S.; Lyu, Y.L.; Liu, L.F.; Yeh, E.T.H. Identification of the molecular basis of doxorubicininduced cardiotoxicity. Nat. Med. 2012, 18, 1639-1642. [CrossRef] [PubMed]

39. Chiong, M.; Wang, Z.V.; Pedrozo, Z.; Cao, D.J.; Troncoso, R.; Ibacache, M.; Criollo, A.; Nemchenko, A.; Hill, J.A.; Lavandero, S. Cardiomyocyte death: Mechanisms and translational implications. Cell. Death Dis. 2011, 2, e244. [CrossRef] [PubMed]

40. Henics, T.; Wheatley, D.N. Cytoplasmic vacuolation, adaptation and cell death: A view on new perspectives and features. Biol. Cell. 1999, 91, 485-498. [CrossRef]

41. Aki, T.; Nara, A.; Uemura, K. Cytoplasmic vacuolization during exposure to drugs and other substances. Cell. Biol. Toxicol. 2012, 28, 125-131. [CrossRef] [PubMed]

42. Shubin, A.V.; Demidyuk, I.V.; Komissarov, A.A.; Rafieva, L.M.; Kostrov, S.V. Cytoplasmic vacuolization in cell death and survival. Oncotarget 2016, 7, 55863-55889. [CrossRef] 